\title{
Vacuum energy in the effective field theory of general relativity. II. Inclusion of fermions and a comment on the QCD contribution
}

\author{
J. Gegelia ${ }^{1,2}$ and Ulf-G. Meißner $\circledast^{3,4,2}$ \\ ${ }^{1}$ Ruhr-University Bochum, Faculty of Physics and Astronomy, Institute for Theoretical Physics II, \\ D-44870 Bochum, Germany \\ ${ }^{2}$ Tbilisi State University, 0186 Tbilisi, Georgia \\ ${ }^{3}$ Helmholtz Institut für Strahlen- und Kernphysik and Bethe Center for Theoretical Physics, \\ Universität Bonn, D-53115 Bonn, Germany \\ ${ }^{4}$ Institute for Advanced Simulation, Institut für Kernphysik and Jülich Center for Hadron Physics, \\ Forschungszentrum Jülich, D-52425 Jülich, Germany
}

(Received 6 September 2019; published 2 December 2019)

\begin{abstract}
Recently, in the framework of a two-loop order calculation for an effective field theory of scalar and vector fields interacting with the metric field, we have shown that for the cosmological constant term which is fixed by the condition of vanishing vacuum energy the graviton remains massless and there exists a selfconsistent effective field theory of general relativity defined on a flat Minkowski background. In the current paper, we extend the two-loop analysis for an effective field theory of fermions interacting with the gravitational field and obtain an analogous result. We also address the issues of fine-tuning of the strong interaction contribution to the vacuum energy and the compatibility of chiral symmetry in the light quark sector with the consistency of the effective field theory of general relativity in a flat Minkovski background.
\end{abstract}

DOI: $10.1103 /$ PhysRevD.100.124002

\section{INTRODUCTION}

It is widely accepted that at low energies the physics of the fundamental particles can be adequately described by effective field theories (EFTs), with the Standard Model being its leading-order approximation [1]. Gravitation can also be included in this framework by considering the effective Lagrangian of metric fields interacting with matter fields [2,3]. Within this approach, the metric field is represented as the Minkowski background plus the graviton field, and the cosmological constant is usually set equal to zero; see, e.g., Ref. [4]. For a nonvanishing cosmological constant term $\Lambda$, the graviton propagator has a pole corresponding to a massive ghost mode [5]. As the cosmological constant term is not suppressed by any symmetry of the effective theory, setting it to zero does not solve the problem because the radiative corrections regenerate the massive ghost [6]. It has been shown in Ref. [6] that one can represent the cosmological constant as a power series in $\hbar$ and adjust the coefficients of this series such that the unphysical mass of the graviton is canceled to all orders in the loop expansion. Thus, to take into account

Published by the American Physical Society under the terms of the Creative Commons Attribution 4.0 International license. Further distribution of this work must maintain attribution to the author(s) and the published article's title, journal citation, and DOI. Funded by SCOAP ${ }^{3}$. a cosmological constant term other than obtained in Ref. [6], it is necessary to consider an EFT in a curved background field. As shown in Ref. [7] by imposing the equations of motion with respect to the nontrivial background graviton field, the mass term of the graviton is removed at tree level. A systematic study of the issue by including the quantum corrections requires an EFT on a curved background metric, which, to the best of our knowledge, is not available yet.

The accelerating expansion of the Universe (see, e.g., Ref. [8] and references therein) leaves us with a huge discrepancy between the measured small value of the effective cosmological constant and its theoretical estimation [9]. In our opinion, if there exists any condition that uniquely fixes the value of the cosmological constant, then it is most natural to expect that it must be imposed by demanding that the energy of the physical vacuum state of the theory describing the Universe is exactly zero. In our recent work [10], we calculated two-loop order contributions of a scalar and vector fields to the vacuum expectation value of the full 4-momentum in a simplified version of the Abelian model with spontaneous symmetry breaking, considered also in Ref. [6]. We found that as a result of a nontrivial cancellation between different diagrams the requirement of vanishing vacuum energy leads to consistency conditions of the considered EFT, first obtained in Ref. [6]. In the current work, we extend this two-loop analysis and calculate the contributions from fermions. 
For the energy-momentum tensor of the gravitational field, we use the definition of the energy-momentum pseudotensor and the full 4-momentum given in the classic textbook by Landau and Lifshitz [11].

Further, in light of the above discussion, we readdress the issue of the fine-tuning of the strong interaction contribution to the vacuum energy and compatibility of the results of Ref. [6] with the chiral symmetry of QCD.

Our work is organized as follows. In Sec. II, we specify the details of the considered EFT of fermions interacting with a gravitational field and calculate one- and two-loop contributions to the vacuum energy and the vacuum expectation value of the gravitational field. In Sec. III, we discuss the QCD contribution to vacuum energy and the problem of the fine-tuning following Ref. [12]. We summarize in Sec. IV, and the Appendix contains the Feynman rules involving fermion fields and two-loop integrals required in our calculations.

\section{VACUUM ENERGY IN AN EFT OF FERMIONS INTERACTING WITH GRAVITONS ON A MINKOWSKI BACKGROUND}

Effective field theory of matter interacting with gravity is described by the most general effective Lagrangian of gravitational and matter fields, which is invariant under general coordinate transformations and the other underlying symmetries,

$$
\begin{aligned}
S & =\int d^{4} x \sqrt{-g}\left\{\mathcal{L}_{\mathrm{gr}}(g)+\mathcal{L}_{\mathrm{m}}(g, \psi)\right\} \\
& =\int d^{4} x \sqrt{-g}\left\{\frac{2}{\kappa^{2}}(R-2 \Lambda)+\mathcal{L}_{\mathrm{gr}, \mathrm{ho}}(g)+\mathcal{L}_{\mathrm{m}}(g, \psi)\right\} \\
& =S_{\mathrm{gr}}(g)+S_{\mathrm{m}}(g, \psi),
\end{aligned}
$$

where $\kappa^{2}=32 \pi G$, with Newton's constant $G=$ $6.70881 \times 10^{-39} \mathrm{GeV}^{-2} ; \psi$ and $g^{\mu \nu}$ denote the matter and metric fields, respectively; $g=\operatorname{det} g^{\mu \nu} ; \Lambda$ is the cosmological constant; and $R$ denotes the scalar curvature. Further, $\mathcal{L}_{\mathrm{m}}(g, \psi)$ is the effective Lagrangian of the matter fields interacting with gravity. Experimental evidence suggests that self-interaction terms of the gravitational field with higher orders of derivatives, represented by $\mathcal{L}_{\text {gr,ho }}(g)$, as well as the nonrenormalizable interactions of $\mathcal{L}_{\mathrm{m}}(g, \psi)$ give contributions to physical quantities which are heavily suppressed for energies accessible by current accelerators. Vielbein tetrad fields have to be introduced for an EFT with fermions.

To be specific, consider the action of the fermions interacting with the gravitational field given by

$$
\begin{aligned}
S_{\mathrm{m}}= & \int d^{4} x \sqrt{-g}\left\{\frac{1}{2} \bar{\psi} i e_{a}^{\mu} \gamma^{a} \nabla_{\mu} \psi-\frac{1}{2} \nabla_{\mu} \bar{\psi} i e_{a}^{\mu} \gamma^{a} \psi-m \bar{\psi} \psi\right\} \\
& +\mathcal{L}_{\mathrm{HO}},
\end{aligned}
$$

where $\mathcal{L}_{\mathrm{HO}}$ denotes the interactions of higher order, the specific form of which is not important for the current work as we will not include them in our calculations. The covariant derivative acting on the fermion field has the form

$$
\begin{aligned}
& \nabla_{\mu} \psi=\partial_{\mu} \psi-\omega_{\mu}^{a b} \sigma_{a b} \psi, \\
& \nabla_{\mu} \bar{\psi}=\partial_{\mu} \bar{\psi}+\bar{\psi} \sigma_{a b} \omega_{\mu}^{a b},
\end{aligned}
$$

where $\sigma_{a b}=\frac{1}{4}\left[\gamma_{a}, \gamma_{b}\right]$ and

$$
\begin{aligned}
& \omega_{\mu}^{a b}=-g^{\nu \lambda} e_{\lambda}^{a}\left(\partial_{\mu} e_{\nu}^{b}-e_{\sigma}^{b} \Gamma_{\mu \nu}^{\sigma}\right) \\
& \Gamma_{\alpha \beta}^{\lambda}=\frac{1}{2} g^{\lambda \sigma}\left(\partial_{\alpha} g_{\beta \sigma}+\partial_{\beta} g_{\alpha \sigma}-\partial_{\sigma} g_{\alpha \beta}\right) .
\end{aligned}
$$

The vielbein fields satisfy the following relations:

$$
\begin{aligned}
e_{\mu}^{a} e_{\nu}^{b} \eta_{a b} & =g_{\mu \nu}, & e_{a}^{\mu} e_{b}^{\nu} \eta^{a b} & =g^{\mu \nu}, \\
e_{\mu}^{a} e_{\nu}^{b} g^{\mu \nu} & =g_{a b}, & e_{a}^{\mu} e_{b}^{\nu} g_{\mu \nu} & =g^{a b} .
\end{aligned}
$$

The energy-momentum tensor corresponding to Eq. (2) has the form [13]

$$
\begin{aligned}
T_{m}^{\mu \nu}= & \frac{i}{4}\left(\bar{\psi} e_{a \mu} \gamma^{a} \nabla_{\nu} \psi+\bar{\psi} e_{a \nu} \gamma^{a} \nabla_{\mu} \psi-\nabla_{\mu} \bar{\psi} e_{a \nu} \gamma^{a} \psi\right. \\
& \left.-\nabla_{\nu} \bar{\psi} e_{a \mu} \gamma^{a} \psi\right)+T_{\mathrm{HO}}^{\mu \nu},
\end{aligned}
$$

where $T_{\mathrm{HO}}^{\mu \nu}$ corresponds to $\mathcal{L}_{\mathrm{HO}}$. Note that we consider one fermion field with mass $m$, the extension to more fermion fields with equal or different masses is straightforward.

For the gravitational field, we have

$$
T_{\mathrm{gr}}^{\mu \nu}(g)=\frac{4}{\kappa^{2}} \Lambda g^{\mu \nu}+T_{L L}^{\mu \nu}(g)
$$

where the pseudotensor $T_{L L}^{\mu \nu}(g)$ is defined via [11]

$$
\begin{aligned}
& (-g) T_{L L}^{\mu \nu}(g)=\frac{2}{\kappa^{2}}\left(\frac{1}{8} g^{\lambda \sigma} g^{\mu \nu} g_{\alpha \gamma} g_{\beta \delta} \mathfrak{g}^{\alpha \gamma}{ }_{\sigma} \mathfrak{g}^{\beta \delta}{ }_{, \lambda}-\frac{1}{4} g^{\mu \lambda} g^{\nu \sigma} g_{\alpha, \gamma} g_{\beta \delta} \mathfrak{g}^{\alpha \gamma}{ }_{\sigma} \mathfrak{g}^{\beta \delta},_{\lambda}-\frac{1}{4} g^{\lambda \sigma} g^{\mu \nu} g_{\beta \alpha} g_{\gamma \delta} \mathfrak{g}^{\alpha \gamma}{ }_{\sigma} \mathfrak{g}^{\beta \delta}{ }_{\lambda}\right. \\
& +\frac{1}{2} g^{\mu \lambda} g^{\nu \sigma} g_{\beta \alpha} g_{\gamma \delta} \mathfrak{g}^{\alpha \gamma}{ }_{\sigma} \mathfrak{g}^{\beta \delta}{ }_{\lambda}+g^{\beta \alpha} g_{\lambda \sigma} \mathfrak{g}^{\nu \sigma}{ }_{\alpha} \mathfrak{g}^{\mu \lambda}{ }_{, \beta}+\frac{1}{2} g^{\mu \nu} g_{\lambda \sigma} \mathfrak{g}^{\lambda \beta}{ }_{, \alpha} \mathfrak{g}^{\alpha \sigma}{ }_{, \beta} \\
& \left.-g^{\mu \lambda} g_{\sigma \beta} \mathfrak{g}^{\nu \beta}{ }_{\alpha} \mathfrak{g}^{\sigma \alpha}{ }_{, \lambda}-g^{\nu \lambda} g_{\sigma \beta} \mathfrak{g}^{\mu \beta},_{\alpha} \mathfrak{g}^{\sigma \alpha}{ }_{\lambda}+\mathfrak{g}^{\lambda \sigma}{ }_{\sigma} \mathfrak{g}^{\mu \nu}{ }_{\lambda}-\mathfrak{g}^{\mu \lambda}{ }_{\lambda} \mathfrak{g}^{\nu \sigma}{ }_{\sigma}\right),
\end{aligned}
$$

with $\mathfrak{g}^{\mu \nu}=\sqrt{-g} g^{\mu \nu}$ and $\mathfrak{g}^{\mu \nu}, \lambda=\partial \mathfrak{g}^{\mu \nu} / \partial x^{\lambda}$. 
From the full energy-momentum tensor $T^{\mu \nu}=$ $T_{\mathrm{m}}^{\mu \nu}(g, \psi)+T_{\mathrm{gr}}^{\mu \nu}(g)$, we obtain the conserved full 4-momentum of the matter and the gravitational field as [11]

$$
P^{\mu}=\int(-g) T^{\mu \nu} d S_{\nu},
$$

where the integration over any hypersurface containing the whole three-dimensional space is implied. Thus, by demanding that the vacuum expectation value of the energy-momentum tensor times $(-g)$ vanishes, we will obtain a vanishing energy of the vacuum. The mentioned vacuum expectation value is given by the path integral

$$
\begin{aligned}
& \left\langle 0\left|(-g) T^{\mu \nu}\right| 0\right\rangle \\
& =\int \mathcal{D} g \mathcal{D} \psi(-g)\left[T_{\mathrm{gr}}^{\mu \nu}(g)+T_{\mathrm{m}}^{\mu \nu}(g, \psi)\right] \\
& \quad \times \exp \left\{i \int d^{4} x \sqrt{-g}\left[\mathcal{L}_{\mathrm{gr}}(g)+\mathcal{L}_{\mathrm{m}}(g, \psi)+\mathcal{L}_{\mathrm{GF}}\right]\right\},
\end{aligned}
$$

where we have added the gauge-fixing term

$$
\mathcal{L}_{\mathrm{GF}}=\xi\left(\partial_{\nu} h^{\mu \nu}-\frac{1}{2} \partial^{\mu} h_{\nu}^{\nu}\right)\left(\partial^{\beta} h_{\mu \beta}-\frac{1}{2} \partial_{\mu} h_{\alpha}^{\alpha}\right),
$$

with $\xi$ the gauge parameter, and the Faddeev-Popov determinant is included in the measure of integration. The condition of vanishing of the right-hand side of Eq. (10) uniquely fixes all coefficients in the power series expansion of the cosmological constant in terms of $\hbar$ :

$$
\Lambda=\sum_{i=0}^{\infty} \hbar^{i} \Lambda_{i}
$$

To perform perturbative calculations, we represent the metric and vielbein fields as sums of the Minkowskian background and the quantum fields $[3,14]$

$$
\begin{aligned}
g_{\mu \nu} & =\eta_{\mu \nu}+\kappa h_{\mu \nu}, \\
g^{\mu \nu} & =\eta^{\mu \nu}-\kappa h^{\mu \nu}+\kappa^{2} h_{\lambda}^{\mu} h^{\lambda \nu}-\kappa^{3} h_{\lambda}^{\mu} h_{\sigma}^{\lambda} h^{\sigma \nu}+\cdots, \\
e_{\mu}^{a} & =\delta_{\mu}^{a}+\frac{\kappa}{2} h_{\mu}^{a}-\frac{\kappa^{2}}{8} h_{\mu \rho} h^{a \rho}+\cdots, \\
e_{a}^{\mu} & =\delta_{a}^{\mu}-\frac{\kappa}{2} h_{a}^{\mu}+\frac{3 \kappa^{2}}{8} h_{a \rho} h^{\mu \rho}+\ldots
\end{aligned}
$$

Applying standard quantum field theory techniques, we obtain the Feynman rules required for the calculations performed here. These are specified in the Appendix when fermion fields are involved (the other ones are given in the Appendix of our earlier paper [10]).

An infinite number of diagrams contribute to the vacuum expectation value of the full energy-momentum pseudotensor times $(-g)$ at tree order; however, all of them vanish if we take $\Lambda_{0}=0$ in Eq. (12) [10]. Notice that this also removes the mass term from the graviton propagator, corresponding to a ghost degree of freedom, at tree order [5].

Next, to obtain the one-loop contributions to the vacuum expectation value of the full energy-momentum pseudotensor times $(-g)$, we calculated the corresponding Feynman diagrams shown in Fig. 2. By demanding that $\Lambda_{1}$ cancels this contribution, we obtain (in the calculations of the loop diagrams below, we applied dimensional regularization, with $d$ the dimension of the space-time, and used the program FEYNCALC $[15,16])$

$$
\Lambda_{1}=\frac{2^{-d} \pi^{-d / 2} \kappa^{2} \mu^{4-d} m^{d} \Gamma\left(1-\frac{d}{2}\right)}{d}
$$

with $\mu$ the scale of dimensional regularization and $\Gamma$ being Euler's $\Gamma$ function. It is a trivial consequence of the definition of the energy-momentum tensor of the matter fields that the same value of $\Lambda_{1}$ cancels the one-loop contribution to the vacuum expectation value of the graviton field $h_{\mu \nu}$, shown in Fig. 1, and, consequently, the graviton self-energy at zero momentum, i.e., graviton mass, as a result of a Ward identity [6]. The first nontrivial result is obtained by calculating the two-loop diagrams contributing to the vacuum expectation value of the full energy-momentum pseudotensor times $(-g)$ shown in Fig. 2 and to the vacuum expectation value of the gravitational field shown in Fig. 1. The same value

$$
\Lambda_{2}=-\frac{2^{-2 d-7} d^{3} \pi^{1-d} \kappa^{4} \mu^{8-2 d} m^{2 d-2} \csc \left(\frac{\pi d}{2}\right) \Gamma\left(-\frac{d}{2}\right)}{(d-2) \Gamma\left(\frac{d}{2}\right)}
$$

cancels both quantities. Here, csc is the cosecans. To check the reliability of the obtained results, we also calculated the two-loop contributions to the graviton self-energy and checked that the same value of $\Lambda_{2}$ ensures that the graviton remains massless in agreement with the Ward identity [6] (we do not give the expressions of the Feynman rules
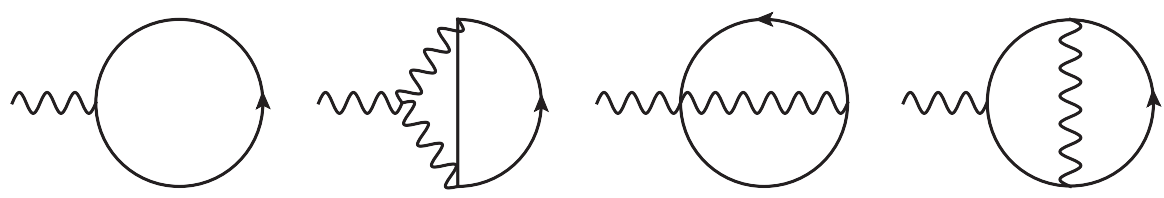

mo

FIG. 1. Diagrams contributing to the vacuum expectation value of the graviton field. The filled circle corresponds to the cosmological constant term. The wiggly and solid lines represent gravitons and fermions, respectively. 

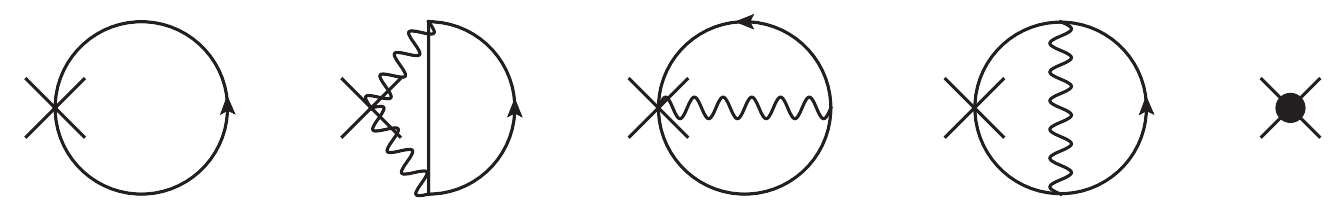

FIG. 2. Diagrams contributing to the vacuum expectation value of the energy-momentum pseudotensor times $(-g)$. The filled circle corresponds to the cosmological constant term. The cross stands for the energy-momentum pseudotensor times $(-g)$, and wiggly and solid lines represent gravitons and fermions, respectively.

needed for the calculation of the graviton self-energy due to their huge size).

\section{QCD CONTRIBUTION TO THE VACUUM ENERGY}

In the framework of general relativity coupled to the Standard Model, the contribution to the cosmological constant originating from the shift in the vacuum energy due to explicit breaking of chiral symmetry of QCD can be calculated with great accuracy [12]. Consider the twoflavor QCD Lagrangian of massless up and down quarks with external scalar and pseudoscalar currents $s(x)$ and $p(x)$, respectively,

$$
\mathcal{L}=-\frac{1}{4} F_{\mu \nu}^{a} F^{a \mu \nu}+i \bar{\psi} \gamma_{\alpha} D^{\alpha} \psi-\bar{\psi}\left(s-i \gamma_{5} p\right) \psi
$$

where $\psi=\left(\psi_{u}, \psi_{d}\right)^{T}$ is a doublet comprising the up and down quark fields. For simplicity, we only consider the two-flavor case here; the extension to three flavors (adding the strange quark) is straightforward. The Lagrangian of Eq. (16) is invariant under $S U(2)_{L} \times S U(2)_{R}$ chiral symmetry transformations

$$
\begin{aligned}
\frac{1}{2}\left(1-\gamma_{5}\right) \psi & \rightarrow L \frac{1}{2}\left(1-\gamma_{5}\right) \psi \\
\frac{1}{2}\left(1+\gamma_{5}\right) \psi & \rightarrow R \frac{1}{2}\left(1+\gamma_{5}\right) \psi \\
(s+i p) & \rightarrow L(s+i p) R^{\dagger}
\end{aligned}
$$

with $L$ and $R$ elements of $S U(2)_{L}$ and $S U(2)_{R}$, respectively. Massless QCD undergoes spontaneous symmetry breaking with pions appearing as Goldstone bosons. The corresponding low-energy effective Lagrangian is given as an expansion in chiral orders, also taking into account the anomaly of the singlet axial current [17]. The lowest-order effective Lagrangian has the form

$$
\mathcal{L}_{2}=\frac{F_{\pi}^{2}}{4} \operatorname{Tr}\left(\partial_{\mu} U \partial^{\mu} U^{\dagger}\right)+\frac{F_{\pi}^{2}}{4} \operatorname{Tr}\left(\chi U^{\dagger}+U \chi^{\dagger}\right),
$$

where $\operatorname{Tr}$ denotes the trace in the flavor (isospin) space and the matrix-valued field $U$ is given in terms of the pion fields $\pi^{a}(a=1,2,3)$ as

$$
U=\exp \left(\frac{i \tau^{a} \pi^{a}}{F_{\pi}}\right)
$$

with $\tau^{a}$ the Pauli matrices and $\chi=2 B_{0}(s+i p)$. Here, $B_{0}$ is a constant of dimension mass related to the vacuum expectation value of the scalar quark condensate, and $F_{\pi}$ is the pion decay constant (in the chiral limit). The Lagrangian of Eq. (18) is invariant under chiral transformations

$$
U \rightarrow L U R^{\dagger}, \quad(s+i p) \rightarrow L(s+i p) R^{\dagger} .
$$

The effective field theory corresponding to QCD is obtained by substituting the external sources as follows:

$$
s=\left[\begin{array}{cc}
m_{u} & 0 \\
0 & m_{d}
\end{array}\right], \quad p=0 .
$$

As the quark masses explicitly break the chiral symmetry, the pions obtain a small mass to leading order in the chiral expansion (much smaller than any other hadron mass)

$$
M_{\pi}^{2}=B_{0}\left(m_{u}+m_{d}\right)+\mathcal{O}\left(m_{q}^{2}\right)
$$

where $m_{q}$ denotes any of the light quark masses. Further, the effective Lagrangian generates a tree-order contribution to the vacuum energy [12]

$$
\Lambda_{m}=-\left\langle 0\left|\mathcal{L}_{2}\right| 0\right\rangle=-F_{\pi}^{2} B_{0}\left(m_{u}+m_{d}\right)=-F_{\pi}^{2} M_{\pi}^{2} .
$$

There is no other term linear in the quark masses in the chiral effective Lagrangian which could compensate the contribution of Eq. (23); e.g., a term like $\operatorname{Tr}\left(\chi+\chi^{\dagger}\right)$ would contribute to the vacuum energy, but it violates the chiral symmetry.

It is argued in Ref. [12] that to cancel the contribution to the vacuum energy given in Eq. (23) one needs to adjust numerically the cosmological constant term in the EFT of pions interacting with gravitation where it is one of the parameters of the effective Lagrangian. Evaluating Eq. (23), one obtains that the chiral symmetry breaking term of QCD gives a large contribution to the vacuum energy

$$
\Lambda_{m}=1.5 \times 10^{8} \mathrm{MeV}^{4}=0.63 \times 10^{43} \Lambda_{\exp },
$$


where $\Lambda_{\text {exp }}=2.4 \times 10^{-47} \mathrm{GeV}^{4}$ is the observed value of the cosmological constant [18]. Reference [12] states, "Because of the large multiplier, if one holds all the other parameters of the Standard Model fixed, a change of the up quark mass in its forty-first digit would produce a change in $\Lambda$ outside the anthropically allowed range. ... Because the calculation is so well controlled, it illustrates the degree of fine-tuning required as well as the futility of thinking that some feature of the Standard Model could lead to a vanishing contribution to $\Lambda$." Below, we critically examine this statement.

It is straightforward to construct a low-energy EFT of pions including the interaction with the gravitational field. The corresponding action has the form

$$
\begin{aligned}
S= & S_{\mathrm{gr}}(g)+\int d^{4} x \sqrt{-g}\left[-F_{\pi}^{2} M_{\pi}^{2}+\frac{1}{2} g^{\mu \nu} \partial_{\mu} \pi^{a} \partial_{\nu} \pi^{a}\right. \\
& \left.-\frac{1}{2} M_{\pi}^{2} \pi^{a} \pi^{a}+\mathcal{O}\left(\pi^{4}\right)\right] .
\end{aligned}
$$

Following the logic of the previous section to cancel the tree-order contribution of the pions to the vacuum energy, we need to take $\Lambda_{0}=F_{\pi}^{2} M_{\pi}^{2}$ in the expansion of Eq. (12). This value of $\Lambda_{0}$ exactly cancels also the graviton mass generated by Eq. (25) at tree order. At one-loop order, there are two diagrams, shown in Fig. 3, contributing to the graviton self-energy generated by the effective Lagrangian of Eq. (25). By demanding that the order $\hbar$ term in Eq. (12) exactly cancels the contribution of these two one-loop diagrams for $p^{2}=0$ ( $p$ is the 4-momentum of the graviton), we obtain

$$
\Lambda_{1}=\frac{3 \kappa^{2}\left(2 M_{\pi}^{2} A_{0}\left(M_{\pi}^{2}\right)+M_{\pi}^{4}\right)}{512 \pi^{2}}
$$

where using dimensional regularization for the loop integral we have

$$
A_{0}\left(M^{2}\right)=\frac{(2 \pi)^{4-d} \mu^{4-d}}{i \pi^{2}} \int \frac{d^{d} k}{k^{2}-M^{2}+i 0^{+}} .
$$

Thus, in full agreement with the results of Ref. [6] to have a self-consistent EFT, the cosmological constant as a parameter of this theory has to be a fixed function of the light quark masses (or equivalently, of the pion
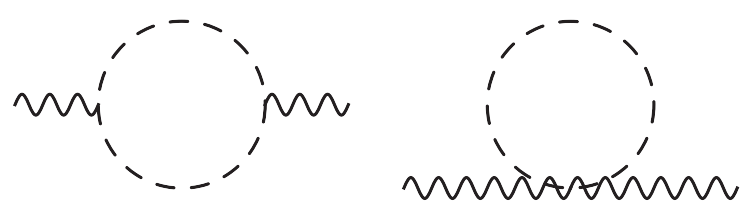

FIG. 3. One-loop diagrams with pions contributing to the graviton self-energy. Wiggly and dashed lines represent gravitons and pions, respectively. mass). This condition imposed on the cosmological constant requires that the cosmological constant term exactly cancels contributions of matter fields to vacuum energy analytically, for any values of the masses and couplings. Such a condition invalidates the considerations of Ref. [12] about the numerical fine-tuning briefly recapitulated above. However, chiral invariance of the low-energy effective Lagrangian of pions interacting with gravitation does not allow a quark-mass-dependent cosmological constant term; see the discussion after Eq. (23).

Thus, on the one hand, the consistency condition of the EFT of general relativity requires that the cosmological constant term is a given fixed function of the light quark masses, and on the other hand, the chiral symmetry of QCD does not allow such a term. The solution to this apparent problem is that the chiral symmetry of the QCD Lagrangian with external sources is not an exact symmetry of the full theory including the gravity.

Let us have a closer look at the action of general relativity given by Eq. (1). According to Ref. [6], the cosmological constant term has to be a fixed function of other parameters of the theory, i.e., $\Lambda \equiv \Lambda\left(m_{u}, m_{d}, g, e, \ldots\right)$, where $e$ is the electromagnetic coupling and the ellipsis stands for other parameters of the effective theory. The Lagrangian $\mathcal{L}_{\mathrm{m}}(g, \psi)$ at leading order coincides with the Lagrangian of the Standard Model, i.e., QCD plus the electroweak theory, taken in a nonflat metric field. To obtain the leading-order Lagrangian of the strong interaction, we "switch off" all other interactions and drop interaction terms with negative mass dimensions (i.e., higher-order "nonrenormalizable" interactions). To switch off gravity, we approximate the metric field $g^{\mu \nu}$ by the constant Minkowski metric, and for the electroweak interaction, we put the corresponding couplings equal to zero. This leaves us with the Lagrangian (for two flavors of quarks)

$\mathcal{L}=-\frac{1}{4} F_{\mu \nu}^{a} F^{a \mu \nu}+\bar{\psi}\left(i \gamma_{\alpha} D^{\alpha}-\mathcal{M}\right) \psi+L_{0}\left(m_{u}, m_{d}, g\right)$,

where we have denoted $-4 \Lambda\left(m_{u}, m_{d}, g, 0,0, \ldots\right) / \kappa^{2}$ by $L_{0}\left(m_{u}, m_{d}, g\right)$. This term does not contradict to any physical symmetries; however, it is not usually included in the QCD Lagrangian because it does not contribute in physical quantities when gravity is not taken into account.

The Lagrangian of Eq. (28) leads to the following contribution to the vacuum energy [19]:

$$
\begin{aligned}
\Lambda_{m} & =\left\langle 0\left|m_{u} \bar{\psi}_{u} \psi_{u}+m_{d} \bar{\psi}_{d} \psi_{d}\right| 0\right\rangle-\left\langle 0\left|L_{0}\left(m_{u}, m_{d}, g\right)\right| 0\right\rangle \\
& =-F_{\pi}^{2} M_{\pi}^{2}-L_{0}\left(m_{u}, m_{d}, g\right) .
\end{aligned}
$$

By taking the yet unspecified constant term of the QCD Lagrangian as $L_{0}\left(m_{u}, m_{d}, g\right)=-F_{\pi}^{2} M_{\pi}^{2}+\mathcal{O}\left(M_{\pi}^{4}\right)=$ $-F_{\pi}^{2} B_{0}\left(m_{u}+m_{d}\right)+\mathcal{O}\left(m_{q}^{2}\right)$ and substituting in Eq. (29), we obtain for the contribution to the vacuum energy $\Lambda_{m}=0+\mathcal{O}\left(m_{q}^{2}\right)$. By adjusting the terms of higher orders 
in light quark masses $m_{q}$ in $L_{0}\left(m_{u}, m_{d}, g\right)$, we can achieve that the QCD contribution to the vacuum energy $\Lambda_{m}$ exactly vanishes for any values of the quark masses.

While canceling the standard QCD contribution to the vacuum energy, the addition of the constant $L_{0}$ term to the Lagrangian does not affect the construction of the lowenergy effective field theory, which proceeds in exact analogy to Ref. [17] by starting with the Lagrangian with external sources

$$
\begin{aligned}
\mathcal{L}_{\mathrm{ext}}= & -\frac{1}{4} F_{\mu \nu}^{a} F^{a \mu \nu}+i \bar{\psi} \gamma_{\alpha} D^{\alpha} \psi+\bar{\psi} \gamma^{\mu}\left(v_{\mu}+a_{\mu} \gamma_{5}\right) \psi \\
& -\bar{\psi}\left(s-i \gamma_{5} p\right) \psi+L_{0}\left(m_{u}, m_{d}, g\right),
\end{aligned}
$$

where the external sources $v_{\mu}(x), a_{\mu}(x), s(x)$, and $p(x)$ are Hermitian, color neutral matrices in flavor space and $s(x)=\mathcal{M}+\cdots$ incorporates the quark mass term. Greens functions of scalar, pseudoscalar, vector, and axial vector currents are generated by the vacuum-to-vacuum transition amplitude

$$
\left\langle 0_{\text {out }} \mid 0_{\text {in }}\right\rangle_{v, a, s, p}=e^{i Z[v, a, s, p]}=\frac{\int \mathcal{D} A \mathcal{D} q e^{i \int d^{4} x \mathcal{L}_{\text {ext }}(x)}}{\int \mathcal{D} A \mathcal{D} q e^{i \int d^{4} x \mathcal{L}(x)}} .
$$

The generating functional $Z[v, a, s, p]$ clearly does not depend on $L_{0}$, and therefore the construction of the lowenergy EFT, namely, chiral perturbation theory, is exactly the same as in Ref. [17], exploiting the fact that the Lagrangian $\mathcal{L}_{\text {ext }}$ without the $L_{0}$ term is invariant under local transformations

$$
\psi(x) \rightarrow\left[\frac{1}{2}\left(1+\gamma_{5}\right) R(x)+\frac{1}{2}\left(1-\gamma_{5}\right) L(x)\right] \psi(x),
$$

provided that the external sources transform as follows:

$$
\begin{aligned}
v_{\mu}^{\prime}+a_{\mu}^{\prime} & =R\left(v_{\mu}+a_{\mu}\right) R^{\dagger}+i R \partial_{\mu} R^{\dagger}, \\
v_{\mu}^{\prime}-a_{\mu}^{\prime} & =L\left(v_{\mu}-a_{\mu}\right) L^{\dagger}+i L \partial_{\mu} L^{\dagger}, \\
s^{\prime}+i p^{\prime} & =R(s+i p) L .
\end{aligned}
$$

We conclude that the presence in the QCD Lagrangian of the $L_{0}\left(m_{u}, m_{d}, g\right)$ term, which is nothing other then a cosmological constant, does not contradict to any physically relevant symmetries of QCD. We remark, again, that these considerations have been performed in Minkowski space-time; a generalization to a curved background has yet to be worked out.

\section{SUMMARY}

By demanding the presence of a massless graviton (instead of a massive spin-2 ghost) in the spectrum of the perturbative EFT of general relativity in flat Minkowski background, the cosmological constant term is uniquely fixed as a function of all other parameters of the theory [6]. We argue that if there is any physical reason for choosing a fixed value of the cosmological constant then it must be the condition of vanishing of the vacuum energy. In our recent paper [10], we calculated the vacuum expectation value of the full 4-momentum of the matter and gravitational fields at two-loop order in a simplified version of the Abelian model with spontaneous symmetry breaking considered also in Ref. [6]. We obtained that as a result of a nontrivial cancellation between different diagrams the requirement of the vanishing vacuum energy leads to the conditions of Ref. [6]. While in Ref. [10] we included only a scalar and vectors as the matter fields, in the current work, we considered the contributions of fermions and obtained similar results. In particular, the value of the cosmological constant, which cancels the two-loop fermion contribution to the vacuum energy, also eliminates the vacuum expectation value of the graviton field and the massive ghost, thus leading to a self-consistent EFT of general relativity in Minkowski background. Being aware of the nonexistence of a commonly accepted expression of the energy-momentum tensor for the gravitational field (see, e.g., Refs. [20-24]), we used the definition of the energy-momentum pseudotensor and the full 4-momentum given in the classic textbook by Landau and Lifshitz [11].

While we are still unable to give a general argument, based on our two-loop order results in an EFT of matter and gravitational fields on flat Minkowski background, we expect that by demanding that the vacuum energy should be vanishing to all orders we obtain a self-consistent perturbative EFT of gravitation coupled to the fields of the Standard Model.

Let us emphasize that for any value of the cosmological constant other than obtained in Ref. [6] it is necessary to consider an EFT in a curved background field. While in this case the mass term of the graviton is removed at tree level [7], a systematic study of the issue by including the quantum corrections, to the best of our knowledge, has not been done due to the lack of corresponding EFT in curved background.

The results of Refs. [6,10] and of the current work resolve the issue of the fine-tuning of the QCD contribution in the vacuum energy addressed in Ref. [12]. In particular, there is no numerical fine-tuning, but rather the cosmological constant, as a function of the parameters of QCD, exactly cancels the QCD contribution to the vacuum energy. However, this solution of the problem seems to be incompatible with the chiral symmetry of QCD. A closer examination, however, reveals that there is no contradiction with any symmetries of QCD with observable physical consequences. Stated differently, it is possible to cancel the QCD contribution to the cosmological constant for any value of the quark masses in a way that does not invalidate the successful use of chiral perturbation theory at low energies. The precise mechanism of this cancellation in 
terms of more fundamental theory underlying the EFT of general relativity coupled to the Standard Model remains to be understood.

\section{ACKNOWLEDGMENTS}

We thank the referee for some clarifying remarks. The work of J. G. was supported in part by BMBF (Grant No. 05P18PCFP1) and by the Georgian Shota Rustaveli National Science Foundation (Grant No. FR17-354). The work of U.-G. M. was supported in part by Deutsche Forschungsgemeinschaft through funds provided to the Sino-German CRC 110 "Symmetries and the Emergence of Structure in QCD" (Grant No. TRR110), by the Chinese Academy of Sciences through a President's International Fellowship Initiative (Grant No. 2018DM0034) and by the VolkswagenStiftung (Grant No. 93562).

\section{APPENDIX: FEYNMAN RULES}

Below, we give Feynman rules involving fermions used in the calculation of the vacuum expectation values of the graviton field and the energy-momentum tensor multiplied with $(-g)$. The other Feynman rules are given in the Appendix of Ref. [10].

Propagators:

(1) Fermion propagator with momentum $p$ :

$$
\frac{i}{\not p-m+i \epsilon} \text {. }
$$

Vertices (all momenta in all vertices are incoming):

(i) Graviton with indices $(\mu, \nu)$-incoming fermion with incoming momentum $p_{1}$ and outgoing fermion with incoming momentum $p_{2}$ :

$$
\begin{aligned}
- & \frac{1}{8} i \kappa\left[2 g^{\mu \nu}\left(2 m-\gamma \cdot p_{1}+\gamma \cdot p_{2}\right)+\gamma^{\mu}\left(p_{1}{ }^{\nu}-p_{2}{ }^{\nu}\right)\right. \\
& \left.+\gamma^{\nu}\left(p_{1}{ }^{\mu}-p_{2}{ }^{\mu}\right)\right] .
\end{aligned}
$$

(ii) Gravitons with (Lorentz indices, momentum) combinations $\left(\mu, \nu, k_{1}\right)$ and $\left(\alpha, \beta, k_{2}\right)$-incoming fermion with incoming momentum $p_{1}$ and outgoing fermion with incoming momentum $p_{2}$ :

$$
\begin{aligned}
& \frac{i \kappa^{2}}{256}\left\{2 \gamma^{\beta} \cdot \gamma^{\mu} \cdot \gamma^{\nu} k_{1}^{\alpha}+2 \gamma^{\beta} \cdot \gamma^{\nu} \cdot \gamma^{\mu}{k_{1}}^{\alpha}-2 \gamma^{\mu} \cdot \gamma^{\nu} \cdot \gamma^{\beta}{k_{1}}^{\alpha}-2 \gamma^{\nu} \cdot \gamma^{\mu} \cdot \gamma^{\beta}{k_{1}}^{\alpha}+2 \gamma^{\alpha} \cdot \gamma^{\mu} \cdot \gamma^{\nu} k_{1}{ }^{\beta}+2 \gamma^{\alpha} \cdot \gamma^{\nu} \cdot \gamma^{\mu} k_{1}{ }^{\beta}\right. \\
& -2 \gamma^{\mu} \cdot \gamma^{\nu} \cdot \gamma^{\alpha} k_{1}{ }^{\beta}-2 \gamma^{\nu} \cdot \gamma^{\mu} \cdot \gamma^{\alpha} k_{1}{ }^{\beta}-2 \gamma^{\alpha} \cdot \gamma^{\beta} \cdot \gamma^{\nu} k_{2}{ }^{\mu}-2 \gamma^{\beta} \cdot \gamma^{\alpha} \cdot \gamma^{\nu} k_{2}{ }^{\mu}+2 \gamma^{\nu} \cdot \gamma^{\alpha} \cdot \gamma^{\beta} k_{2}{ }^{\mu}+2 \gamma^{\nu} \cdot \gamma^{\beta} \cdot \gamma^{\alpha} k_{2}{ }^{\mu} \\
& -2 \gamma^{\alpha} \cdot \gamma^{\beta} \cdot \gamma^{\mu} k_{2}{ }^{\nu}-2 \gamma^{\beta} \cdot \gamma^{\alpha} \cdot \gamma^{\mu} k_{2}{ }^{\nu}+2 \gamma^{\mu} \cdot \gamma^{\alpha} \cdot \gamma^{\beta} k_{2}{ }^{\nu}+2 \gamma^{\mu} \cdot \gamma^{\beta} \cdot \gamma^{\alpha} k_{2}{ }^{\nu}+4 \gamma^{\mu} \cdot \gamma^{\nu} \cdot\left(\gamma \cdot k_{1}\right) g^{\alpha \beta}+4 \gamma^{\nu} \cdot \gamma^{\mu} \cdot\left(\gamma \cdot k_{1}\right) g^{\alpha \beta} \\
& -4\left(\gamma \cdot k_{1}\right) \cdot \gamma^{\mu} \cdot \gamma^{\nu} g^{\alpha \beta}-4\left(\gamma \cdot k_{1}\right) \cdot \gamma^{\nu} \cdot \gamma^{\mu} g^{\alpha \beta}-3 \gamma^{\beta} \cdot \gamma^{\nu} \cdot\left(\gamma \cdot k_{1}\right) g^{\alpha \mu}-\gamma^{\beta} \cdot \gamma^{\nu} \cdot\left(\gamma \cdot k_{2}\right) g^{\alpha \mu}-\gamma^{\nu} \cdot \gamma^{\beta} \cdot\left(\gamma \cdot k_{1}\right) g^{\alpha \mu} \\
& -3 \gamma^{\nu} \cdot \gamma^{\beta} \cdot\left(\gamma \cdot k_{2}\right) g^{\alpha \mu}+\left(\gamma \cdot k_{1}\right) \cdot \gamma^{\beta} \cdot \gamma^{\nu} g^{\alpha \mu}+3\left(\gamma \cdot k_{1}\right) \cdot \gamma^{\nu} \cdot \gamma^{\beta} g^{\alpha \mu}+3\left(\gamma \cdot k_{2}\right) \cdot \gamma^{\beta} \cdot \gamma^{\nu} g^{\alpha \mu}+\left(\gamma \cdot k_{2}\right) \cdot \gamma^{\nu} \cdot \gamma^{\beta} g^{\alpha \mu} \\
& -3 \gamma^{\beta} \cdot \gamma^{\mu} \cdot\left(\gamma \cdot k_{1}\right) g^{\alpha \nu}-\gamma^{\beta} \cdot \gamma^{\mu} \cdot\left(\gamma \cdot k_{2}\right) g^{\alpha \nu}-\gamma^{\mu} \cdot \gamma^{\beta} \cdot\left(\gamma \cdot k_{1}\right) g^{\alpha \nu}-3 \gamma^{\mu} \cdot \gamma^{\beta} \cdot\left(\gamma \cdot k_{2}\right) g^{\alpha \nu}+\left(\gamma \cdot k_{1}\right) \cdot \gamma^{\beta} \cdot \gamma^{\mu} g^{\alpha \nu} \\
& +3\left(\gamma \cdot k_{1}\right) \cdot \gamma^{\mu} \cdot \gamma^{\beta} g^{\alpha \nu}+3\left(\gamma \cdot k_{2}\right) \cdot \gamma^{\beta} \cdot \gamma^{\mu} g^{\alpha \nu}+\left(\gamma \cdot k_{2}\right) \cdot \gamma^{\mu} \cdot \gamma^{\beta} g^{\alpha \nu}-3 \gamma^{\alpha} \cdot \gamma^{\nu} \cdot\left(\gamma \cdot k_{1}\right) g^{\beta \mu}-\gamma^{\alpha} \cdot \gamma^{\nu} \cdot\left(\gamma \cdot k_{2}\right) g^{\beta \mu} \\
& -\gamma^{\nu} \cdot \gamma^{\alpha} \cdot\left(\gamma \cdot k_{1}\right) g^{\beta \mu}-3 \gamma^{\nu} \cdot \gamma^{\alpha} \cdot\left(\gamma \cdot k_{2}\right) g^{\beta \mu}+\left(\gamma \cdot k_{1}\right) \cdot \gamma^{\alpha} \cdot \gamma^{\nu} g^{\beta \mu}+3\left(\gamma \cdot k_{1}\right) \cdot \gamma^{\nu} \cdot \gamma^{\alpha} g^{\beta \mu}+3\left(\gamma \cdot k_{2}\right) \cdot \gamma^{\alpha} \cdot \gamma^{\nu} g^{\beta \mu} \\
& +\left(\gamma \cdot k_{2}\right) \cdot \gamma^{\nu} \cdot \gamma^{\alpha} g^{\beta \mu}-3 \gamma^{\alpha} \cdot \gamma^{\mu} \cdot\left(\gamma \cdot k_{1}\right) g^{\beta \nu}-\gamma^{\alpha} \cdot \gamma^{\mu} \cdot\left(\gamma \cdot k_{2}\right) g^{\beta \nu}-\gamma^{\mu} \cdot \gamma^{\alpha} \cdot\left(\gamma \cdot k_{1}\right) g^{\beta \nu}-3 \gamma^{\mu} \cdot \gamma^{\alpha} \cdot\left(\gamma \cdot k_{2}\right) g^{\beta \nu} \\
& +\left(\gamma \cdot k_{1}\right) \cdot \gamma^{\alpha} \cdot \gamma^{\mu} g^{\beta \nu}+3\left(\gamma \cdot k_{1}\right) \cdot \gamma^{\mu} \cdot \gamma^{\alpha} g^{\beta \nu}+3\left(\gamma \cdot k_{2}\right) \cdot \gamma^{\alpha} \cdot \gamma^{\mu} g^{\beta \nu}+\left(\gamma \cdot k_{2}\right) \cdot \gamma^{\mu} \cdot \gamma^{\alpha} g^{\beta \nu}+4 \gamma^{\alpha} \cdot \gamma^{\beta} \cdot\left(\gamma \cdot k_{2}\right) g^{\mu \nu} \\
& +4 \gamma^{\beta} \cdot \gamma^{\alpha} \cdot\left(\gamma \cdot k_{2}\right) g^{\mu \nu}-4\left(\gamma \cdot k_{2}\right) \cdot \gamma^{\alpha} \cdot \gamma^{\beta} g^{\mu \nu}-4\left(\gamma \cdot k_{2}\right) \cdot \gamma^{\beta} \cdot \gamma^{\alpha} g^{\mu \nu}+4\left(-4 p_{1}{ }^{\nu} \gamma^{\mu} g^{\alpha \beta}+4 p_{2}{ }^{\nu} \gamma^{\mu} g^{\alpha \beta}\right. \\
& -4 p_{1}{ }^{\mu} \gamma^{\nu} g^{\alpha \beta}+4 p_{2}{ }^{\mu} \gamma^{\nu} g^{\alpha \beta}-16 m g^{\mu \nu} g^{\alpha \beta}+8 \gamma \cdot p_{1} g^{\mu \nu} g^{\alpha \beta}-8 \gamma \cdot p_{2} g^{\mu \nu} g^{\alpha \beta}+3 p_{1}{ }^{\beta} \gamma^{\nu} g^{\alpha \mu}-3 p_{2}{ }^{\beta} \gamma^{\nu} g^{\alpha \mu} \\
& +3 p_{1}{ }^{\beta} \gamma^{\mu} g^{\alpha \nu}-3 p_{2}{ }^{\beta} \gamma^{\mu} g^{\alpha \nu}+\left(3\left(p_{1}{ }^{\nu}-p_{2}{ }^{\nu}\right) \gamma^{\alpha}+3\left({p_{1}}^{\alpha}-p_{2}{ }^{\alpha}\right) \gamma^{\nu}+8\left(2 m-\gamma \cdot p_{1}+\gamma \cdot p_{2}\right) g^{\alpha \nu}\right) g^{\beta \mu} \\
& +3 p_{1}{ }^{\mu} \gamma^{\alpha} g^{\beta \nu}-3 p_{2}{ }^{\mu} \gamma^{\alpha} g^{\beta \nu}+3 p_{1}{ }^{\alpha} \gamma^{\mu} g^{\beta \nu}-3 p_{2}{ }^{\alpha} \gamma^{\mu} g^{\beta \nu}+16 m g^{\alpha \mu} g^{\beta \nu}-8 \gamma \cdot p_{1} g^{\alpha \mu} g^{\beta \nu}+8 \gamma \cdot p_{2} g^{\alpha \mu} g^{\beta \nu} \\
& \left.\left.-4 p_{1}{ }^{\beta} \gamma^{\alpha} g^{\mu \nu}+4 p_{2}{ }^{\beta} \gamma^{\alpha} g^{\mu \nu}+\gamma^{\beta}\left(3 p_{1}{ }^{\nu} g^{\alpha \mu}-3 p_{2}{ }^{\nu} g^{\alpha \mu}+3 p_{1}{ }^{\mu} g^{\alpha \nu}-3 p_{2}{ }^{\mu} g^{\alpha \nu}-4 p_{1}{ }^{\alpha} g^{\mu \nu}+4 p_{2}{ }^{\alpha} g^{\mu \nu}\right)\right)\right\} \text {. }
\end{aligned}
$$

(iii) Energy-momentum tensor with indices $(\mu, \nu)$ incoming fermion with incoming momentum $p_{1}$ and outgoing fermion with incoming momentum $p_{2}$ :

$$
\frac{1}{2}\left(\gamma^{\mu} p_{1}^{\nu}+\gamma^{\nu} p_{1}^{\mu}\right)
$$

(iv) Energy-momentum tensor with indices $(\mu, \nu)-$ graviton with (Lorentz indices, momentum) combination $\left(\alpha, \beta, k_{1}\right)$-incoming fermion with incoming momentum $p_{1}$ and outgoing fermion with incoming momentum $p_{2}$ : 


$$
\begin{aligned}
\frac{1}{32} & \kappa\left\{-\gamma^{\beta} \cdot\left(\gamma \cdot k_{1}\right) \cdot \gamma^{\nu} g^{\alpha \mu}-\gamma^{\nu} \cdot \gamma^{\beta} \cdot\left(\gamma \cdot k_{1}\right) g^{\alpha \mu}+\gamma^{\nu} \cdot\left(\gamma \cdot k_{1}\right) \cdot \gamma^{\beta} g^{\alpha \mu}+\left(\gamma \cdot k_{1}\right) \cdot \gamma^{\beta} \cdot \gamma^{\nu} g^{\alpha \mu}-\gamma^{\beta} \cdot\left(\gamma \cdot k_{1}\right) \cdot \gamma^{\mu} g^{\alpha \nu}\right. \\
& -\gamma^{\mu} \cdot \gamma^{\beta} \cdot\left(\gamma \cdot k_{1}\right) g^{\alpha \nu}+\gamma^{\mu} \cdot\left(\gamma \cdot k_{1}\right) \cdot \gamma^{\beta} g^{\alpha \nu}+\left(\gamma \cdot k_{1}\right) \cdot \gamma^{\beta} \cdot \gamma^{\mu} g^{\alpha \nu}-\gamma^{\alpha} \cdot\left(\gamma \cdot k_{1}\right) \cdot \gamma^{\nu} g^{\beta \mu}-\gamma^{\nu} \cdot \gamma^{\alpha} \cdot\left(\gamma \cdot k_{1}\right) g^{\beta \mu} \\
& +\gamma^{\nu} \cdot\left(\gamma \cdot k_{1}\right) \cdot \gamma^{\alpha} g^{\beta \mu}+\left(\gamma \cdot k_{1}\right) \cdot \gamma^{\alpha} \cdot \gamma^{\nu} g^{\beta \mu}-\gamma^{\alpha} \cdot\left(\gamma \cdot k_{1}\right) \cdot \gamma^{\mu} g^{\beta \nu}-\gamma^{\mu} \cdot \gamma^{\alpha} \cdot\left(\gamma \cdot k_{1}\right) g^{\beta \nu}+\gamma^{\mu} \cdot\left(\gamma \cdot k_{1}\right) \cdot \gamma^{\alpha} g^{\beta \nu} \\
& +\left(\gamma \cdot k_{1}\right) \cdot \gamma^{\alpha} \cdot \gamma^{\mu} g^{\beta \nu}-2\left(\gamma^{\beta} g^{\alpha \nu} p_{1}{ }^{\mu}+\gamma^{\alpha} g^{\beta \nu} p_{1}{ }^{\mu}-\gamma^{\beta} g^{\alpha \nu} p_{2}{ }^{\mu}-\gamma^{\alpha} g^{\beta \nu} p_{2}{ }^{\mu}+2 \gamma^{\nu}\left(p_{1}{ }^{\alpha} g^{\beta \mu}-p_{2}{ }^{\alpha} g^{\beta \mu}+g^{\alpha \mu} p_{1}{ }^{\beta}\right.\right. \\
& \left.-g^{\alpha \mu} p_{2}{ }^{\beta}-2 g^{\alpha \beta} p_{1}{ }^{\mu}+2 g^{\alpha \beta} p_{2}{ }^{\mu}\right)+\gamma^{\beta} g^{\alpha \mu} p_{1}{ }^{\nu}+\gamma^{\alpha} g^{\beta \mu} p_{1}{ }^{\nu}-\gamma^{\beta} g^{\alpha \mu} p_{2}{ }^{\nu}-\gamma^{\alpha} g^{\beta \mu} p_{2}{ }^{\nu} \\
& \left.\left.+2 \gamma^{\mu}\left(p_{1}{ }^{\alpha} g^{\beta \nu}-p_{2}{ }^{\alpha} g^{\beta \nu}+g^{\alpha \nu} p_{1}{ }^{\beta}-g^{\alpha \nu} p_{2}{ }^{\beta}-2 g^{\alpha \beta} p_{1}{ }^{\nu}+2 g^{\alpha \beta} p_{2}{ }^{\nu}\right)\right)\right\} .
\end{aligned}
$$

The two-loop master integral appearing in the results of various two-loop calculations is

$$
\begin{aligned}
\int \frac{d^{d} k_{1} d^{d} k_{2}}{(2 \pi)^{2 d}} \frac{1}{\left(k_{1}^{2}-M^{2}+i \epsilon\right)^{\alpha}\left(k_{2}^{2}-M^{2}+i \epsilon\right)^{\beta}\left(\left(k_{1}-k_{2}\right)^{2}+i \epsilon\right)^{\gamma}} \\
=\frac{i^{2-2 \alpha-2 \beta-2 \gamma} M^{2(d-\alpha-\beta-\gamma)} \Gamma\left(\frac{d}{2}-\gamma\right) \Gamma\left(\alpha+\gamma-\frac{d}{2}\right) \Gamma\left(\beta+\gamma-\frac{d}{2}\right) \Gamma(\alpha+\beta+\gamma-d)}{(4 \pi)^{d} \Gamma(\alpha) \Gamma(\beta) \Gamma\left(\frac{d}{2}\right) \Gamma(\alpha+\beta+2 \gamma-d)} .
\end{aligned}
$$

[1] S. Weinberg, The Quantum Theory of Fields (Cambridge University Press, Cambridge, England, 2005), Vols. 1-2.

[2] J. F. Donoghue, Phys. Rev. D 50, 3874 (1994).

[3] J. F. Donoghue and B. R. Holstein, J. Phys. G 42, 103102 (2015).

[4] J. F. Donoghue, M. M. Ivanov, and A. Shkerin, arXiv: 1702.00319 .

[5] M. J. G. Veltman, Conf. Proc. C 7507281, 265 (1975).

[6] D. Burns and A. Pilaftsis, Phys. Rev. D 91, 064047 (2015).

[7] G. Gabadadze and A. Gruzinov, Phys. Rev. D 72, 124007 (2005).

[8] D. Rubin and B. Hayden, Astrophys. J. 833, L30 (2016).

[9] S. Weinberg, Rev. Mod. Phys. 61, 1 (1989).

[10] J. Gegelia and U.-G. Meißner, Phys. Rev. D 100, 046021 (2019).

[11] L. D. Landau and E. M. Lifshitz, The Classical Theory of Fields (Pergamon Press, Oxford, 1975).

[12] J. F. Donoghue, Annu. Rev. Nucl. Part. Sci. 66, 1 (2016).

[13] N. D. Birrell and P.C.W. Davies, Quantum Fields in Curved Space (Cambridge University Press, Cambridge, England, 1984).
[14] G. 't Hooft and M. J. G. Veltman, Ann. Inst. H. Poincare Phys. Theor. A 20, 69 (1974).

[15] R. Mertig, M. Bohm, and A. Denner, Comput. Phys. Commun. 64, 345 (1991).

[16] V. Shtabovenko, R. Mertig, and F. Orellana, Comput. Phys. Commun. 207, 432 (2016).

[17] J. Gasser and H. Leutwyler, Ann. Phys. (N.Y.) 158, 142 (1984).

[18] M. Tanabashi et al. (Particle Data Group), Phys. Rev. D 98, 030001 (2018).

[19] J. F. Donoghue, E. Golowich, and B. R. Holstein, Dynamics of the Standard Model, 2nd ed. (Cambridge University Press, Cambridge, England, 2014).

[20] S. V. Babak and L. P. Grishchuk, Phys. Rev. D 61, 024038 (1999).

[21] L. M. Butcher, A. Lasenby, and M. Hobson, Phys. Rev. D 78, 064034 (2008).

[22] L. B. Szabados, Living Rev. Relativity 12, 4 (2009).

[23] L. M. Butcher, M. Hobson, and A. Lasenby, Phys. Rev. D 82, 104040 (2010).

[24] L. M. Butcher, M. Hobson, and A. Lasenby, Phys. Rev. D 86, 084013 (2012). 